\title{
NMR INVESTIGATIONS OF PHTHALOCYANINE
}

\author{
B. DUDREVA and S. GRANDE
}

\author{
Sektion Physik, Karl-Marx-University, Leipzig
}

\begin{abstract}
Résumé. - Au cours d'investigations systématiques des semi-conducteurs du type phtalocyanine, Rosenstein et ses collaborateurs ont trouvé que la forme $\alpha$ de la phtalocyanine sans métal $\left(\mathrm{H}_{2} \mathrm{Pc}\right)$ présente une anomalie diélectrique vers la température de $17^{\circ} \mathrm{C}$ pour les très faibles taux de refroidissement. Ils suggèrent que la substance subit un changement de phase vers l'état ferroélectrique.

Par la méthode NMR, nous essayons de poursuivre l'étude du mécanisme de la transition de phase, dans la poudre de $\mathrm{H}_{2} \mathrm{Pc}$. Nous avons mesuré le temps $T_{1}$ de relaxation spin-réseau des protons dans l'intervalle de température allant de $-140^{\circ} \mathrm{C}$ à $+150^{\circ} \mathrm{C}$. Les résultats dépendent sensiblement de la préparation de l'échantillon. La dépendance en température du temps de relaxation peut être expliquée par deux mécanismes de relaxation. Une légère anomalie de $T_{1}$ à la transition de phase peut être observée. En vue d'ultérieures interprétations de la dynamique, nous avons aussi mesuré $T_{1}$ pour la phtalocyanine deutérée $\left(\mathrm{D}_{2} \mathrm{Pc}\right)$. On donne une discussion des résultats.
\end{abstract}

\begin{abstract}
In the course of systematic investigations of the organic semiconductors of the phthalocyanine type, Rosenstein and coworkers have found that the $\alpha$-form of the metalfree Phthalocyanine $\left(\mathrm{H}_{2} \mathrm{Pc}\right)$ shows an anomaly in the dielectric behaviour at a temperature of about $17^{\circ} \mathrm{C}$ for very small cooling rates. They suggested that the substance undergoes a phase transition to the ferroelectric state.

With the NMR-method we try to investigate the mechanism of the phase transition in the powder of $\mathrm{H}_{2} \mathrm{Pc}$ further. We have measured the spin-lattice relaxation time $T_{1}$ of the protons in the temperature-range from $-140^{\circ} \mathrm{C}$ to $+150^{\circ} \mathrm{C}$. The results depend sensitively on the preparation of the sample. The temperature dependence on the relaxation time can be explained by two relaxation mechanisms. A small anomaly of $T_{1}$ at the phase transition can be observed. For further interpretations of the dynamics we have also measured $T_{1}$ of the deuterated Phthalocyanine $\left(\mathrm{D}_{2} \mathrm{Pc}\right)$. The results have been discussed.
\end{abstract}

1. Structure and molecular properties. - Phthalocyanine $(\mathrm{Pc})$ has been studied by different physical methods for its interesting properties. First of all, its semiconductive properties are of great significance, as well as its colouring effect.

The structure of the plane $\mathrm{H}_{2} \mathrm{Pc}$ molecule is shown in figure 1. The inner Porphyrin ring is also of great importance in the structure of many biological molecules. A given percentage of $\pi$-bonds is found in this ring and in the other bonds, too. There are two hydrogen bridges in the inside of the ring of the metalfree $\mathrm{Pc}$, which are responsible for a slight deformation of the symmetrical complex.

The metalfree Pc crystallizes in three modifications $\alpha, \beta$ and $\gamma$ according to the way of production (Ebert, Hamann $[4,5])$. The $\beta$ type is the most stable one. The crystalline structure has been studied by Robertson [1]. The elementary cell corsists of two molecules, whose planes are inclined at an angle of about $45^{\circ}$ with respect to the $b$-axis. The minimum intermolecular distance is approximately $3,5 \AA$.

Optical investigations on powdered samples, layers and monocrystals (Heilmeier, Chadderton [2], [3], [6]) give nearly the same results. For the semiconducting properties it was possible to determine from these results the band gaps and conducting mechanism due to excitation of $\pi$-bonds. The structure of the spectra

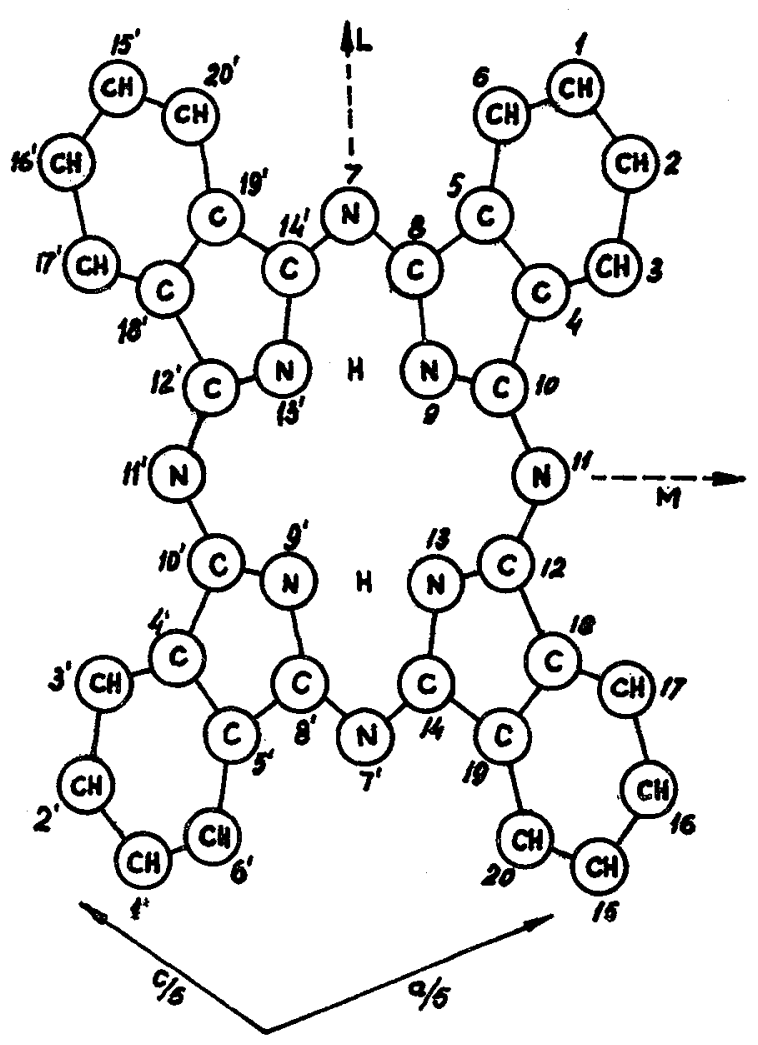

FIG. 1, - Structure of the $\mathrm{H}_{2} \mathrm{Pc}$ molecule in the b-direction. 
suggests the existence of a vibration at $395 \mathrm{~cm}^{-1}$, which is supposed to result from a torsion motion of the whole molecule.

2. Ferroelectric properties. - The results of dielectric measurements on layers of $\mathrm{H}_{2} \mathrm{Pc}$ were first published in 1969 by Rosenstein [7]-[11]. They show anomalies in the temperature dependence similar to these in ferroelectric substances. The temperature at which anomalies occur is between $17^{\circ} \mathrm{C}$ and $20^{\circ} \mathrm{C}$. Anomalous behaviour has been found only for a slow cooling rate. Other quantities show anomalities, too the conductivity for example rises by $1-2$ orders compared with the other Pc-s. Thermal hysteresis was found as well. This points out that the structural rearrangement in the region of the phase transition and occurrence or disappearance of the spontaneous polarization are accompanied by a variation of the excitation energy of the current carriers.

In the case of the sample of interest $\mathrm{H}_{2} \mathrm{Pc}$, the so-called ferroelectric absorption has also been studied. It is expressed by a slow drop of the current with time. This effect is due to the re-orientation of the domains.

Under the influence of weak fields, the curve current against voltage of the ferroelectric phase $\mathrm{H}_{2} \mathrm{Pc}$ becomes non-linear near the transition temperature.

The frequency and temperature dependencies of the dielectric behaviour make it possible to conclude that a dielectric relaxation takes place. Unlike SbSJ, no shift of the transition temperature is found during illumination, but a change of the dielectric constant.

We have also measured the temperature dependence of the dielectric constant $\varepsilon$ in the range from $-50^{\circ} \mathrm{C}$ to $+70^{\circ} \mathrm{C}$ for polycrystalline samples. Figure 2 shows the results for a tablet produced by a pressure of $2000 \mathrm{kp} / \mathrm{cm}^{2}$, which demonstrates an anomaly at the phase transition temperature.

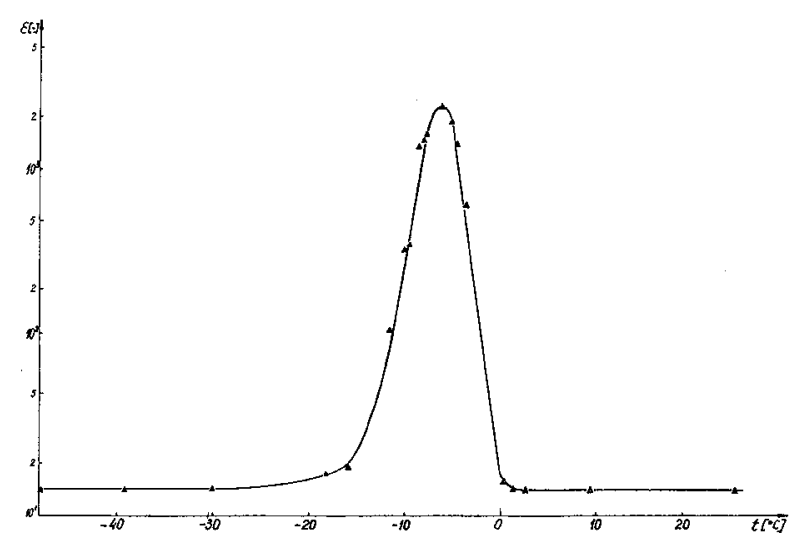

FIG. 2. - Temperature dependence of $\varepsilon$ for a tablet of $\mathrm{H}_{2} \mathrm{Pc}$ (probe III) at $25 \mathrm{kHz}$.

3. NMR investigations. - NMR investigations were carried out in order to provide a closer look at the mechanism of the phase transition in $\mathrm{H}_{2} \mathrm{Pc}$. The spin-lattice relaxation time $T_{1}$ of protons was measured in powdered $\mathrm{H}_{2} \mathrm{Pc}$ in the temperature range of $-140^{\circ} \mathrm{C}$ to $150^{\circ} \mathrm{C}$ by the help of the $\pi / 2-\pi / 2$ impulse method at $32 \mathrm{MHz}$, employing a Bruker impulse spectrometer. All measurements were performed on a dark sample, in order to avoid the photosensitivity of $\mathrm{H}_{2} \mathrm{Pc}$. Since the transition of $\mathrm{H}_{2} \mathrm{Pc}$ to the low temperature phase is a very slow process, all $T_{1}$ curves were taken during heating. In order of eleminating any influence of the sample's state, the temperature dependence of three different probes has been measured. Sample I is technically pure (crude). Sample II was purified in $\mathrm{H}_{2}$ stream at $300^{\circ} \mathrm{C}$, during 8 hours, due to the catalytic effect (Calvin [12], [13]). The oxygen presence in the material was reduced by this method. Sample III was produced by a double sublimation in a vacuum of $10^{-2}$ torr. Infra-red absorption measurements show that the sample I is a mixture of $\alpha$ and $\beta$ type, $\alpha$ type being prevalent. The sample II is in the $\beta$ modification. The sample III is again a mixture of predominant $\alpha$ type. In the sample IV the two protons forming the $\mathrm{H}$-bond in the centre of the molecule have been replaced for $98 \%$ due to deuterium. Figure 3 shows the $T_{1}$ curves

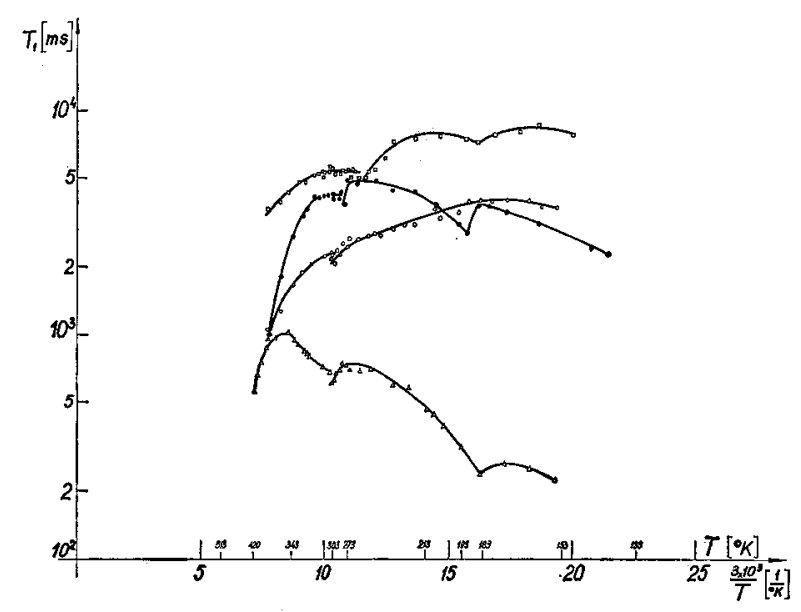

FIG. 3. - Temperature dependence of the proton spin-lattice relaxation time $T_{1}$ at $32 \mathrm{MHz}: \Delta$ : Sample I, technicaly pure ; - : Sample II-Sample I,purified in $\mathrm{H}_{2}$; $\mathrm{O}$ : Sample III, double sublimatied $\mathrm{H}_{2} \mathrm{Pc} ; \square$ : Sample IV, $\mathrm{D}_{2} \mathrm{Pc}$.

plotted against $1 / T$. All samples including the deuterated one show a similar temperature behaviour. As we can see, the relaxation times in our substances are firstly determined by the interaction with the paramagnetic centres. According to the results of Assour [14] the concentration of free radicals in the technical $\mathrm{H}_{2} \mathrm{Pc}$ is nearly $10^{17} \mathrm{~cm}^{-3}$. Thus, a comparatively small relaxation times of the sample $I$ is observed. The contents of oxygen and consequently the concentration of the free radicals are evidently reduced by treating the material with $\mathrm{H}_{2}$. Owing to this, the relaxation times increase by one order, though still under the influence of their interaction with the electron spins. All substances show an anomaly at $T_{\mathrm{c}}$ in the relaxation behaviour, which we relate to the particular dielectric behaviour. The relaxation above $T_{\mathbf{c}}$ 
corresponds to the normal values for the diffusion in $\mathrm{H}$-bonds and explains the smaller relaxation rate of the deuterated substance at high temperatures.

Since the relaxation times of these sample is higher at lower temperatures too, we assume a relaxation mechanism due to the exchange of protons in the $\mathrm{H}$-bonds in the some molecule. We get

$$
\frac{1}{T_{1 \text { meas }}}=\frac{N_{\mathrm{H}}}{N_{\mathrm{all}}} \cdot \frac{1}{T_{1 \mathrm{H}}},
$$

where

$$
T_{1 \mathrm{H}}=\frac{1}{9 T_{1 \text { meas }}} .
$$

The relaxation due to the paramagnetic centres which always exists should be nearly the same also in the deuterated sample.
The anomalies of $T_{1}$ at the temperature of the phase transition can be explained by a additional relaxation due to a torsional motion of the whole molecule. There may be exists an electric dipol moment caused by a nonsymmetrical distribution of $\mathrm{H}$-atoms in the centre of the molecule.

Yet, nothing can be said about the second peculiarity at $-75^{\circ} \mathrm{C}$ and its relationship with the structural parameters.

The large temperature dependence of the relaxation time of all samples above room temperature car be explained by diffusion of protons amiong the molecules of the substance. The potential barriere is estimated to $E=6.6 \mathrm{kcal} / \mathrm{mol}$ according to a simple relaxation expression, suggested by Blombergen, Purcell, Pound (See Abragam).

\section{References}

[1] Robertson (J.), J. Chem. Soc., 1936, 1195.

[2] Heilmeier (G.), J. Chem. Phys., 1963, 38, 893.

[3] Heilmeier (G.), J. Chem. Phys., 1963, 38, 897.

[4] Ebert (A.), J. Am. Chem. Soc., 1952, 74, 2806.

[5] Hamann (C.), Phys. Stat. Sol., 1964, 4, 509.

[6] Chadderton (L.), J. Phys. Chem. Sol., 1963, 24, 751.
[7-11] Rosenstein (L.), FTT, 1969, 11, 2403, 2695, 3383. 1970, 12, 634, 2772.

[12] Calvin, Trans. Far. Soc., 1936, 32, 1443.

[13] Calvin, Trans. Far. Soc., 1936, 32, 1436.

[14] Assour (J.), J. Chem. Phys., 1964, 872. 